\title{
Integrated Current-Mode Spatial Filtering Detector with Variable Multi-valued Weighting Function
}

\author{
Hyunmin Go Non-member (SAMSUNG TECHWIN CO., LTD., dawn6767@arazio.net) \\ Jun-ya Takayama Non-member (Tokyo Institute of Technology, takayama@ctrl.titech.ac.jp) \\ Shinji Ohyama Member (Tokyo Institute of Technology, ohyama@kuramae.ne.jp) \\ Akira Kobayashi Member (Tokyo Institute of Technology, akobaakoba2005@yahoo.co.jp)
}

Keywords: Integrated Spatial Filtering Detector with Variable Multi-valued Weighting Function, Spatial Filtering Method, Spatial Filtering Detector, Multiplying Digital-to-Analog Converter, Weighted-sum Structure

The spatial filtering method(SFM) based on imagevelocity sensing has often been used in the velocity measurement. The SFM can be expressed as

$$
g(x, y ; t)=\int_{-\infty}^{\infty} \int_{-\infty}^{\infty} f(x, y ; t) h(x, y) d x d y
$$

where $h(x, y)$ is the weighting function of the spatial filter and $f(x, y ; t)$ is the spatial pattern of the object. Adaptively designing the weighting function $h(x, y)$, the effective characteristics of the spatial pattern of the object appear as an output of the spatial filtering detector(SFD). The conventional SFDs, however, have disadvantages in that the weighting function $h(x, y)$ is fixed. In accordance with the optical pattern of the object, it is necessary to change the weighting function to obtain the desired information.

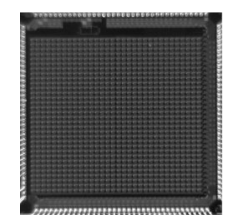

(a) Prototype of ISFD-VMWF

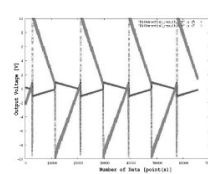

(b) Differential output signals @ $\mathrm{G}=100,1000[\mathrm{kV} / \mathrm{A}]$
Fig. 1. ISFD-VMWF and differential output signals

In this paper, designed and fabricated novel ISFD-VMWF which has $32 \times 32$ weighted pixel elements(WPE), 7-bit signed analog weighting circuit and digital weighting control circuit using the weighted-sum structure. In the weighted structure, the 7-bit signed analog weighting circuit is constructed by multiplying digital-to-analog converter using cascode current mirrors. The weights are controlled and programmmed by external interface through the PC. Achieving the sum structure, the weighted current from each WPE is summed into one current signal, and two output signals, plus and minus, are outputted. These signals are converted to the voltage signal and amplified by the op-amp with various gains. Figure 1 (a) shows the fabricated ISFD-VMWF. As a basic characteristic of ISFD-VMWF, Fig. 1 (b) depicts acquired outputs at gains $100[\mathrm{kV} / \mathrm{A}]$ and $1000[\mathrm{kV} / \mathrm{A}]$ with shifting the weighting values from -63 to 63 , on total pixels at the same time. At this test, weight changing speed is 23.4 [ms].

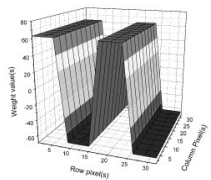

(a) 2-split weighting function

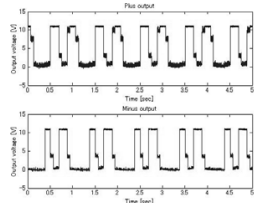

(b) Its plus/minus output signals
Fig. 2. Output signals by adopting an evalution weighting function

In order to examine the validity of the ISFD-VMWF, an experiment of the simple velocity measurement is executed. When the weighting function is divided with 2 , 4 , and 8 split, multiplied frequency ratio according to the reference frequecy is depited in Fig. 3. From these results, the ISFD-

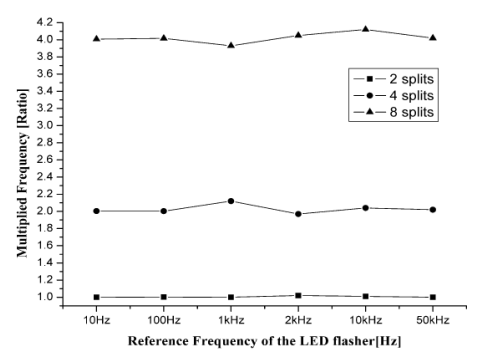

Fig. 3. Velocity measurement results

VMWF is able to measure the velocity of over $0.5[\mathrm{~mm} / \mathrm{sec}]$, which is same as the 2000 [frames/sec] of the digital imager. The cutoff frequency of the ISFD-VMWF is 200 [kHz]. 


\section{Integrated Current-Mode Spatial Filtering Detector with Variable Multi-valued Weighting Function}

$\begin{array}{ll}\text { Hyunmin Go* } & \text { Non-member } \\ \text { Jun-ya Takayama** } & \text { Non-member } \\ \text { Shinji Ohyama** }^{*} & \text { Member } \\ \text { Akira Kobayashi** } & \text { Member }\end{array}$

In this paper, we designed and fabricated a new Integrated Spatial Filtering Detector with Variable Multivalued Weighting Function (ISFD-VMWF). This chip based on 32x32 array of n-well/p-sub photodiodes has been successfully developed using the analog/digital mixed implementation. The weighted-sum structure is proposed to realize the ISFD-VMWF. As the weighted structure, the 7-bit signed analog weighting circuit is used with cascode current mirrors. The weighting control partis consisted of digital registers in a weighted pixel element as the sum structure. The weights are controlled and programmed by external interface on the PC. Achieving the sum structure, the weighted current from each processing cell element is summed into one current signal, and two output signals, plus and minus, are outputted. These signals are converted to the voltage signal and amplified by the external differential op-amp with various gains with parallel. The experimental results show that output signals summed up are linearly shifted with changing weights as the basic characteristics of the ISFD-VMWF. To evaluate the ISFD-VMWF, another experiment of the velocity measurement was executed, which shows the effectiveness of the ISFD-VMWF on the measurement system.

Keywords: Integrated Spatial Filtering Detector, Spatial Filtering Method, Multiplying Digital-to-Analog Converter, Current-Mode Weighted-sum Structure, Spatial Parallel Processing

\section{Introduction}

The spatial filtering method (SFM) based on imagevelocity sensing has often been used in the velocity measurement ${ }^{(1)-(4)}$. In addition to spatial-filtering velocimetry, many algorithms in the decade have been proposed such as a road surface recognition sensor ${ }^{(5)}$, moment analysis $^{(6)}$ and so forth ${ }^{(7)}$. These proposed algorithms and techniques use the advantages of the SFM which are the simplicity of its optical and mechnical setup, and high-speed processing based on the spatial parallel processing. The SFM can be expressed by the spatial integral as

$$
g(x, y ; t)=\int_{-\infty}^{\infty} \int_{-\infty}^{\infty} f(x, y ; t) h(x, y) d x d y
$$

where $h(x, y)$ is the weighting function of the spatial filter and $f(x, y ; t)$ is the spatial pattern of the object. In the SFM, the role of the spatial weighting function $h(x, y)$ determines the characteristics of the spatial filtering detector by the shape of $h(x, y)$. Adaptively designing the weighting function $h(x, y)$, the effective characteristics of the spatial pattern of the object appear as

\footnotetext{
* SAMSUNG TECHWIN CO., LTD.

145-3 Sandaewon 1-Dong, Jungwon-Gu, Sungnam-city, Kyungki-Do, 462-703 KOREA

** Tokyo Institute of Technology

2-12-1, O-okayama, Meguro-ku, Tokyo 152-8552
}

output of the spatial filtering detector. These conventional spatial filtering detectors (SFD), however, have disadvantages in that the weighting function $h(x, y)$ is fixed and the weighting values are binary or ternary values. In accordance with the optical pattern of the object, it is necessary to adjust the weighting function to acquire the desired information, eliminating the effect of the disturbance and constructing a more accurate measurement system ${ }^{(8)}$. In Uddin's paper, an adaptive method can be the most accurate because of its ability to adjust the filter parameters so as to tune the spatial filters to the stochastic characteristics of the object ${ }^{(4)}$. To realize the adaptive method and the optimum measurement in the SFM, the spatial filter must have the ability to adjust the filter parameters by regulating the correlation length of the surface pattern of the object and the slit width of the spatial filter.

Besides establishing an accurate measurement system, programmable weighting functions are required for executing various applications. The arrow-feathers-type SFD was proposed for obtaining the two-dimensional velocity vector ${ }^{(9)}$, the radial-type SFD for detecting the rotational speed ${ }^{(10)}$. Introduced SFDs are fabricated with application specified hardware configuration, so that these structure were impossible to change and adjust to the measuring objective.

A method to solve this problem and to achieve high performance with SFM is to change the parameter of the spatial filter according to the object. Changing pa- 
rameters means the weighting function can be changeable. In addition, many more weighting functions that is continuously changeable and easily set have been required to achieve the active and adaptive measurement system. To overcome these disadvantages, We proposed a new type of spatial filtering detector, called the VSFD. This SFD has variable multi-valued weighting function with $16 \times 16$ photodiode array ${ }^{(11)}$. Although the VSFD has discretely exchangeable 8-bit signed weighting values, the system is big in size of about $45 \mathrm{~cm} \mathrm{x} 35 \mathrm{~cm}$. Moreover, the handmade photodiode array of the VSFD decreases the measurement accuracy. From these considerations, the integration of the VSFD becomes one of a current issue of our research.

As a solution of these problems above described, we present a new current-mode integrated SFD with variable multi-valued weighting function (ISFD-VMWF) in this paper. This ISFD-VMWF is a device that can exchange programmably weighting functions and 7-bit signed weighting values for adaptive measurement. Furthermore, this integration produces advanced results that are the miniaturization of the SFD and low power consumption in comparison with the presented SFDs. Plus and minus signed output signals from the ISFDVMWF are analog and continuous. We fabricate the ISFD-VMWF chip using the CMOS $0.35 \mu \mathrm{m}$ process through VDEC and show the test results that output signals are linearly shifted with changing weights as a basic characteristics of the ISFD-VMWF.

\section{Realization of the ISFD-VMWF}

It is difficult that the weighted-sum structure using $\mathrm{R}-2 \mathrm{R}$ ladder circuitry introduced from the reference is integrated as it is by CMOS standard process ${ }^{(6)}$. That because the photo-current from a photodiode is not suitable so weakly as to integrate on a chip using the currentdivision method by the R-2R circuitry. On-resistance of the FET switch and the difference of $\mathrm{R}$ affect the current-division ratio and occur serious error of the precision on the VSFD. To realize the proposed weightedsum structure in the integration technology, we adopt current-multiplication-type weighted-sum structure.

2.1 The Weighted-sum Structure The small region is correspondent to the pixel on the image plane in the case of image processing. That is to say, the realization of the variable multi-valued weighting function is a pixel with multiple weighting values that can be varied discretely in this case ${ }^{(12)}$.

There are two methods to change the signal value. The first method is to use the effect of multiplication by the elements or configuration with the amplifying function. The second one is to use the effect of the addition with the superposition function such as the condensing lens and the time integral. We propose a weighted-sum structure to realize variable multi-valued weighting function in small region in the ISFD-VMWF.

Each small region has the weighted structure and the total of them are constructed by the sum structure. To achieve this structure in the circuitry, an adopted method in this paper is as follows:
[Weight structure]

Current-mode MDAC

[Sum Structure]

Current-mode summation by the single-node connection of all pixel outputs

\section{Configuration of ISFD-VMWF}

Figure 1 shows the system configuration of the ISFDVMWF chip. The ISFD-VMWF chip consists of $32 \times 32$ photodiode array, weighting circuits with 7-bit signed weighting values using current-mode parallel multiplying digital to analog converter (MDAC), weight control circuit by D flip-flop and differential op-amp. The specification of the ISFD-VMWF chip is summarized in Table 1.

3.1 Current-mode Weighting Circuit To realize the weighted-sum structure on the ISFD-VMWF, it is necessary for signals to be summed at final output terminals. The more weighted pixel elements are in the ISFD-VMWF chip, the more many nodes to be summed are required.

These multiple summation of signals from the

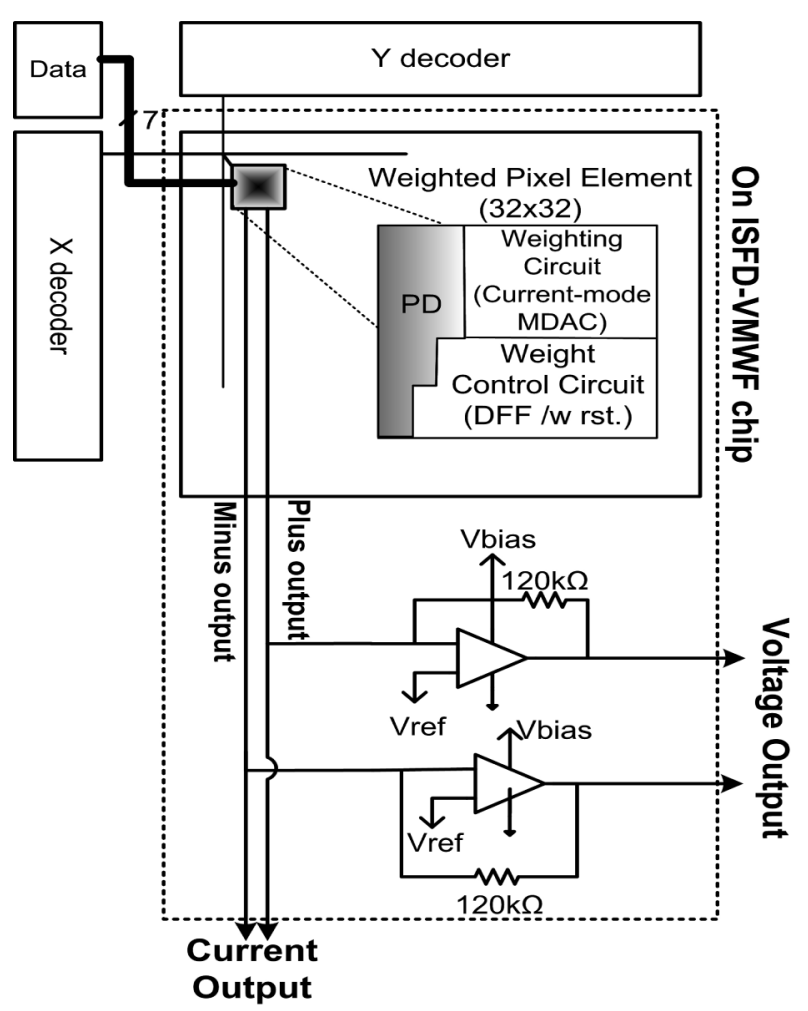

Fig. 1. Configuration of ISFD-VMWF chip

Table 1. Specification of ISFD-VMWF chip

\begin{tabular}{ll}
\hline process & CMOS $0.35 \mu \mathrm{m} \mathrm{2-Poly} \mathrm{3-Metal} \mathrm{(VDEC)}$ \\
pixel area & $2424 \mu \mathrm{m}^{2}$ \\
photodetector & n-well/p-sub PD with guard ring \\
weighting method & current-mode MDAC \\
weighting values & 7 -signed bits \\
WPE size & $116.5 \mu \mathrm{m} \times 98 \mu \mathrm{m}$ \\
fill factor & $10.74 \%$ \\
operating voltage & $3.3 \mathrm{~V}$ \\
chip size & $4.8 \times 4.8 \mathrm{~mm}^{2}$ \\
package & QFP 160 \\
\hline
\end{tabular}


weighted pixel elements (WPE) enables the currentmode sum structure where the number of signal nodes can be added by only wiring the nodes. There are two advantages of this structure; one is that the error by the mismatch of MOS device is reduced as long as the driving circuits have a high output resistance. Another is that current signals are less influenced by the noise in comparison with the voltage signals and have negligible loss in wiring.

While the sum structure is possibly performed by wiring, in the weighted structure, the weighting can be compactly achieved by current multiplication with current mirrors. With the use of two-fold cascode current mirrors, less error originated from the circuits in addition higher output resistance desired than a current mirror. Therefore, the weighted-sum structure can be successfully realized by current-mode circuitry.

3.2 Weighted Pixel Element When the light is incident upon the PD, the generated photocurrent is parallelly mirrored to the output signal line through the cascode current mirrors. The current ratio of the photocurrent to output current signals of current mirrors in the weighting circuit is determined by the dimension ratio of MOS devices.

The circuit shown in Fig. 2 is a CMOS current-mode 6-bit MDAC ${ }^{(13)}$. In this design, current gains are designed from 0 to 63 to achieve weighting the input signal and controlled by switching on/off through external in-

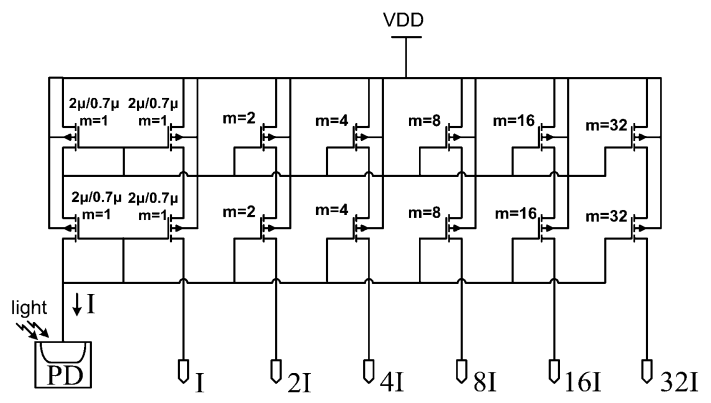

Fig. 2. Current-mode weighting circuit

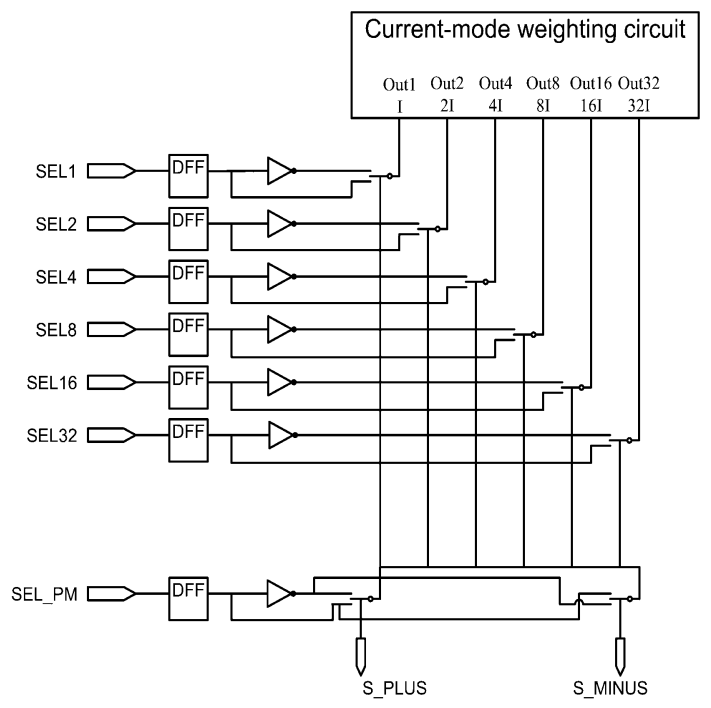

Fig. 3. Weighting control circuit terface. The output of each MDAC is connected to one of two summing nodes, plus/minus signal nodes, according to the sign bit associated with the weighted signal. Figure 3 shows the schematic of the weighting control circuit. A layout of the overall circuit of the WPE is shown in Fig. 4. The photodiode is surrounded by a guard ring which prevents it from the sensitive analog circuits as well as from coupling effects through the psub. Moreover, the photodiode is separated from the registers. Metal 1 and 2 layers are used for routing circuit. Metal 3 layer is partially used for routing, but is mainly used for shielding light except the photodiode array region not to generate parasitic photo-carriers and partially for routing.

3.3 Operational Amplifier In this ISFD-VMWF chip, we use a general 2-stage operational amplifier structure and a bias circuit ${ }^{(14)}$. A bias circuit of op-amp is insensitive to the temperature in order to decrease the effect of the rising temperature by the illumination. The differential amplifiers are established at both sides of output current signals in order to convert to voltage signals through $120[\mathrm{k} \Omega]$. The gain of the amplifier is about $63 \mathrm{~dB}$, and phase margin is 62 degrees ${ }^{(14)}$.

3.4 Simulation and Photograph We report a simulation result using $\mathrm{HSPICE}^{\dagger}$ when the photocurrent $I_{p d}$ is mirrored by current mirrors from 0 to 63 times by

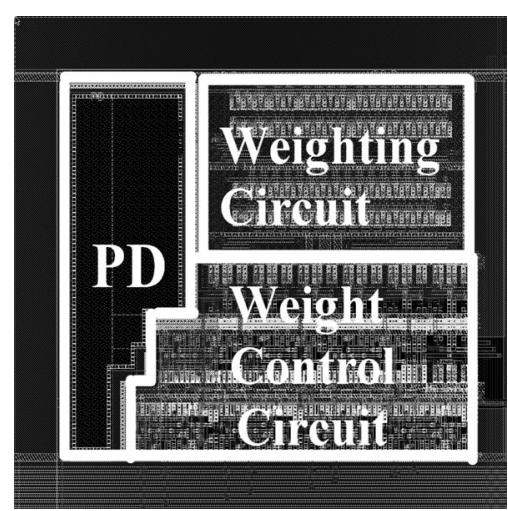

Fig. 4. WPE layout

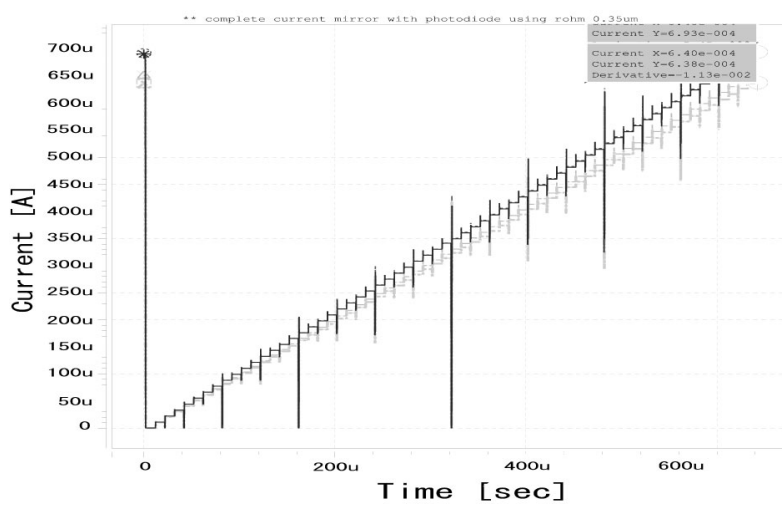

Fig. 5. Output current at the weighting circuit @ $I_{p d}=20[\mathrm{nA}]$

$\dagger$ This work is supported by VLSI Design and Education Center(VDEC), the University of Tokyo in collaboration with Synopsys, Inc. 


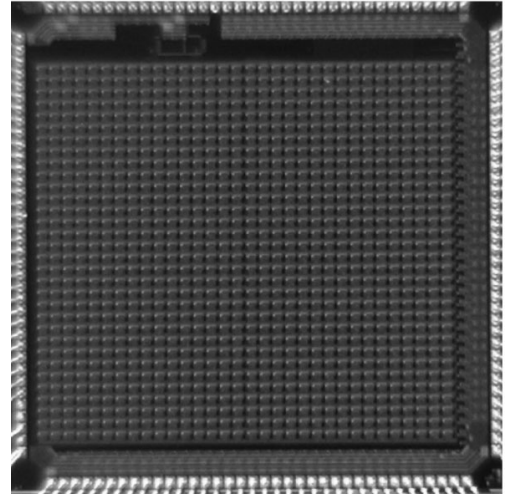

Fig. 6. A photograph of the fabricated ISFD-VMWF chip

switch on/off operations as shown in Fig. 2 and Fig. 3. Figure 5 shows the output current at an end node of the weighting circuit with changing three MOS - slow , typical, and fast - model when the photocurrent is $20[\mathrm{nA}]$. Apart from other than the result shown in this paper, sufficient simulation with exchanging $I_{p d}$ has been performed. The gain error of mirrored current is within about $6[\%]$ when fast MOS model is used.

Figure 6 shows a photograph of the fabricated ISFDVMWF chip using CMOS $0.35 \mu \mathrm{m}$ process with 2-poly 3 -metal layers through VDEC.

\section{Experiment}

4.1 Experimental Setup Figure 7 shows the experimental measurement system implementing an ISFD-VMWF chip. The control and measurement computer installing a 8-bit digital input/output board and a 12-bit analog input/output board control the ISFDVMWF. At this time, we use output current signals from the ISFD-VMWF chip. Output current signals are converted to voltage signals amplified to $1[\mathrm{kV} / \mathrm{A}]$, $10[\mathrm{kV} / \mathrm{A}], 100[\mathrm{kV} / \mathrm{A}]$, and $1000[\mathrm{kV} / \mathrm{A}]$ gains by the external differential op-amp circuits within the ISFDVMWF measurement system. The gains, 1, 10, 100 and $1000[\mathrm{kV} / \mathrm{A}]$ of the differential op-amp can be controlled by the control program through the analog-to-digital conversion board.

4.2 Basic Characteristics To ascertain the output characteristics of the ISFD-VMWF, tests are operated by changing the weighting values from -63 to 63,127 steps, on total pixels at the same time. The output voltage of plus signal is shown in Fig. 8 (a) using $1[\mathrm{k} \Omega]$ resistance. Figure $8(\mathrm{~b})$ shows acquired raw data of differential output signals of plus and minus signals at the gain $100[\mathrm{kV} / \mathrm{A}]$ (small wave) and $1000[\mathrm{kV} / \mathrm{A}]$ (big wave) for $3 \frac{1}{2}$ periods under about 1100 [lux] $(-2.06 \mathrm{dBm} / \mathrm{W}$ optical power $)$. In this test, weight changing speed is about 23.4 [msec] that is dependent on the clock of the control computer and the specification of the digital input/output board. Assuming that the driving circuit using FPGA or H8 is applied, weight changing speed can be under 1 [ms].

Figure 9 (a) shows output signals are linearly varied with exchanging weights on all pixels as a basic output characteristic of the ISFD-VMWF. The errors due to

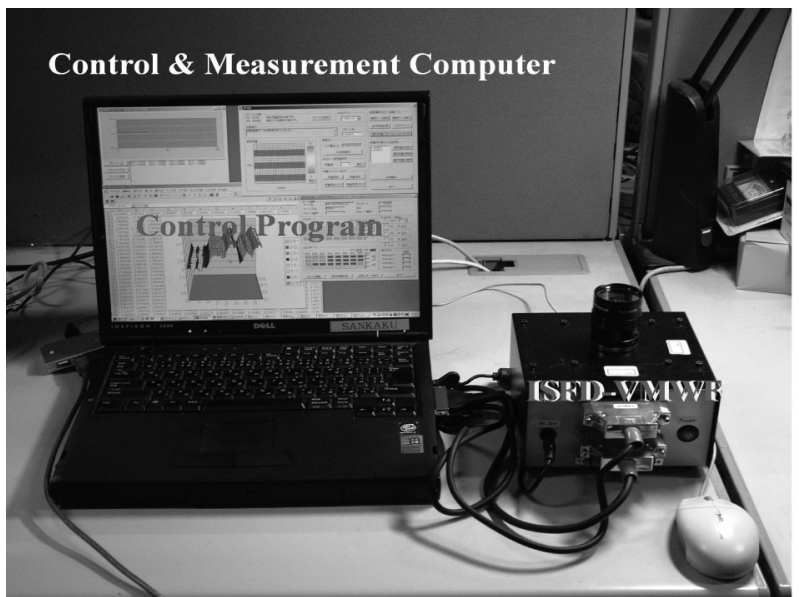

Fig. 7. Experimental measurement system

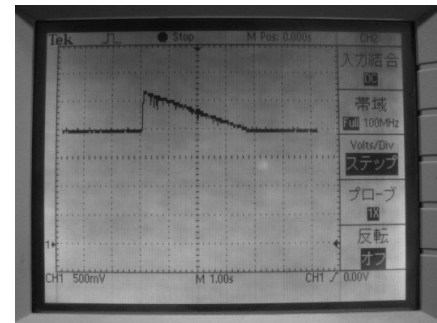

(a) Plus side output signal

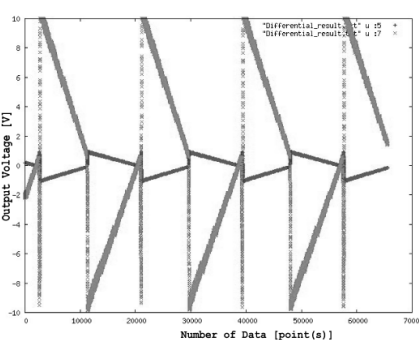

(b) Differential output signals @ G $=100,1000[\mathrm{kV} / \mathrm{A}]$
Fig. 8. Differential output signals

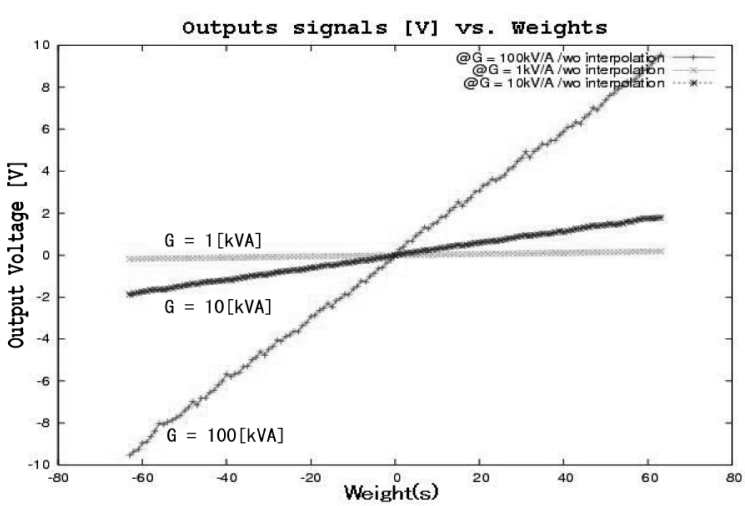

(a) Basic characteristics of the ISFD-VMWF @ $\mathrm{G}=1,10,100[\mathrm{kV} / \mathrm{A}]$

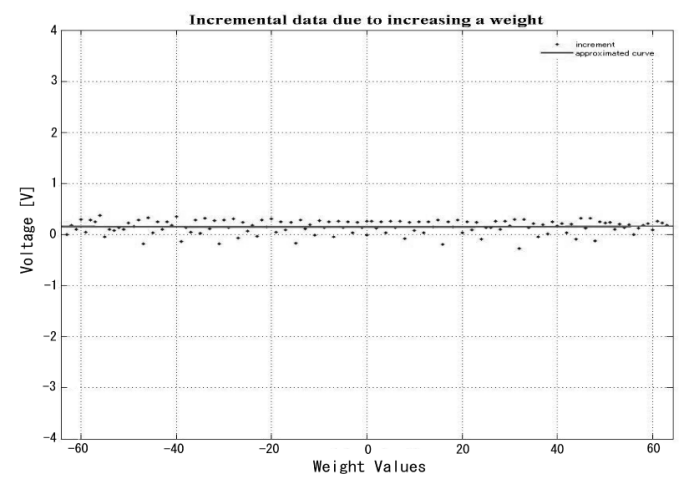

(b) Error due to increasing a weight value

Fig. 9. Basic characteristics of the ISFD 


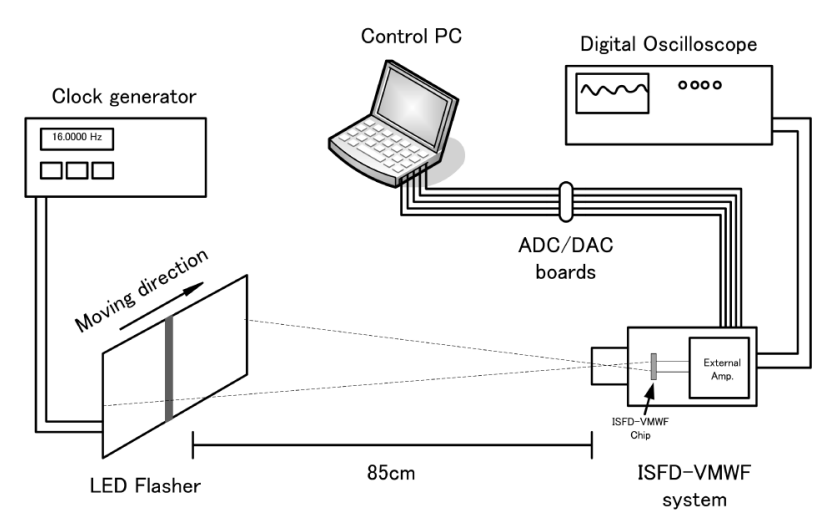

Fig. 10. Scheme of the velocity measurement system using the ISFD-VMWF

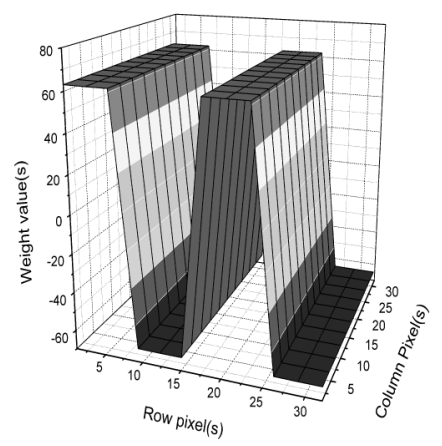

(a) A weighting function to evaluate plus/minus outputs
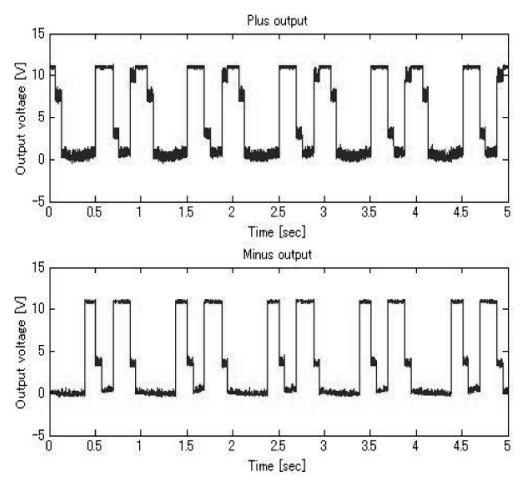

(b) Output signals by adopting the evaluation weighting function

Fig. 11. Output signals by adopting an evalution weighting function

shifting up a weight value at various gains are with $5 \%$ as shown in Fig. 9 (b).

4.3 An Experiment on Velocity Measurement To confirm the validity of the ISFD-VMWF in the application, we manufacture a simple velocity measurement system composed of the LED flasher, the control PC, the ISFD-VMWF, the clock generator, and digital oscilloscope as shown in Fig.10. The LED flasher are made of 16 lines moving along the $\mathrm{x}$ coordinate by impressing clock frequency. This means that a line of LED flasher are moving at $1 / 16$ speed of the clock. Figure 11 shows output signals of plus and minus in Fig.11(b) on the weight function 11 (a).

Table 2 shows an experimental result under the condition of splitting the weighting function of the ISFD-
Table 2. Velocity measurement results

\begin{tabular}{rrrr}
\hline & 2 splits & 4 splits & 8 splits \\
\hline $10[\mathrm{~Hz}]$ & $10.00[\mathrm{~Hz}]$ & $20.04[\mathrm{~Hz}]$ & $40.08[\mathrm{~Hz}]$ \\
$100[\mathrm{~Hz}]$ & $100.13[\mathrm{~Hz}]$ & $200.30[\mathrm{~Hz}]$ & $401.64[\mathrm{~Hz}]$ \\
$1[\mathrm{kHz}]$ & $1.00[\mathrm{kHz}]$ & $2.12[\mathrm{kHz}]$ & $3.93[\mathrm{kHz}]$ \\
$2[\mathrm{kHz}]$ & $2.03[\mathrm{kHz}]$ & $3.93[\mathrm{kHz}]$ & $8.09[\mathrm{kHz}]$ \\
$10[\mathrm{kHz}]$ & $10.01[\mathrm{kHz}]$ & $20.04[\mathrm{kHz}]$ & $40.12[\mathrm{kHz}]$ \\
$50[\mathrm{kHz}]$ & $50.00[\mathrm{kHz}]$ & $100.01[\mathrm{kHz}]$ & $200.01[\mathrm{kHz}]$ \\
\hline
\end{tabular}

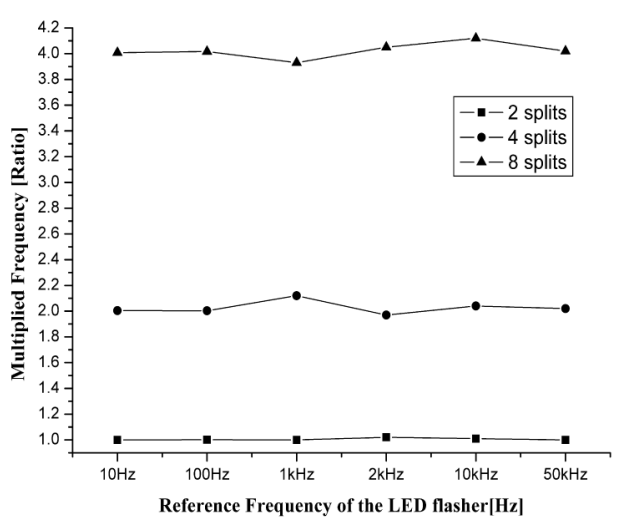

Fig. 12. Velocity measurement results

VMWF. Multiplied ratio according to the reference frequecy of the LED flasher is depited in Fig. 12. Figure 13 shows the velocity experimental results under the LED moving speed $0.5[\mathrm{~mm} / \mathrm{sec}]$ : (a) plot a wave on 2-divided 62 plus and -62 minus weight values setting, namely 16 columns of the WPE of the ISFD-VMWF was set to 62 weight values and other 16 columns set to -62 weight values. (c) is divided 4 regions and (e) is divided 8 sections as the same method of (a) . Regardless of the same moving speed, the ISFD-VMWF can split the wave of 4 times, which means the frequency are 4 times of the clock. In this figure, in spite of the saturation of some waves caused by the fixed step gains of the external amplification stage, the results show that the frequency of the output signal is in proportion to the moving speed of the LED flasher. To avoid the saturation effect, the adjustment of the bias of the amplifier is required and considered in a future work that configure variable external amplification gains.

From these results, the ISFD-VMWF is able to measure the velocity of over $0.5[\mathrm{~mm} / \mathrm{sec}]$, which is same as the 2000 [frames/sec] of the digital imager. The cutoff frequency of the ISFD-VMWF is $200[\mathrm{kHz}]$.

\section{Conclusion}

In this paper, we have designed and fabricated a new current-mode integrated spatial filtering detector with variable multi-valued weighting function (ISFDVMWF) for adaptive measurement. To realize the ISFD-VMWF, the proposed current-mode weightedsum structure is used. The current-mode parallel MDAC, digital memories, and differential op-amp were adopted. The fabricated ISFD-VMWF has 7-bit signed weighting values and various weighting function by external interface. The experiment results show output signals are linearly shifted with exchanging weights on all pixels as a basic output characteristic of the ISFD- 

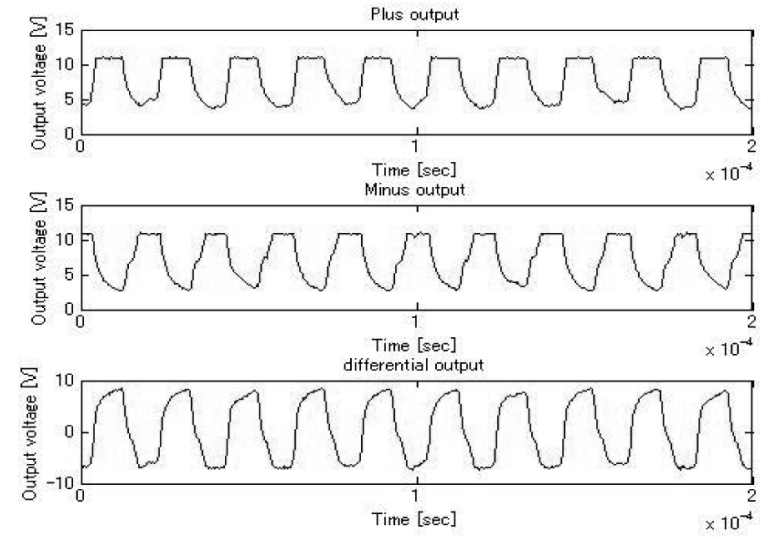

(a) Output wave under the plus/minus 2-divided weighting function along the $\mathrm{x}$ coordinate

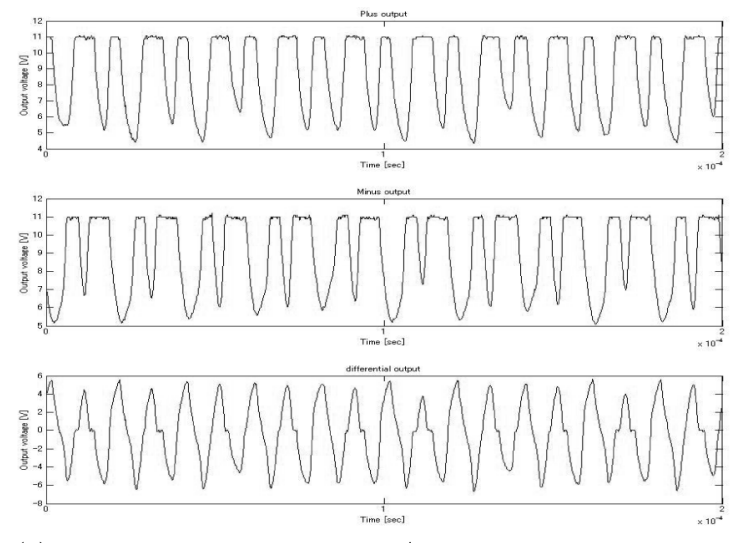

(c) Output wave under the plus/minus 8-divided weighting function along the $\mathrm{x}$ coordinate

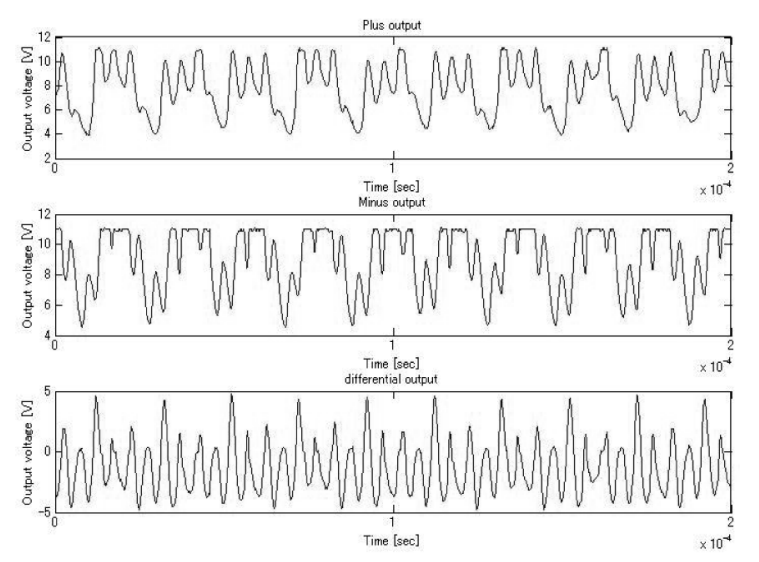

(e) Output wave under the plus/minus 4-divided weighting function along the $\mathrm{x}$ coordinate

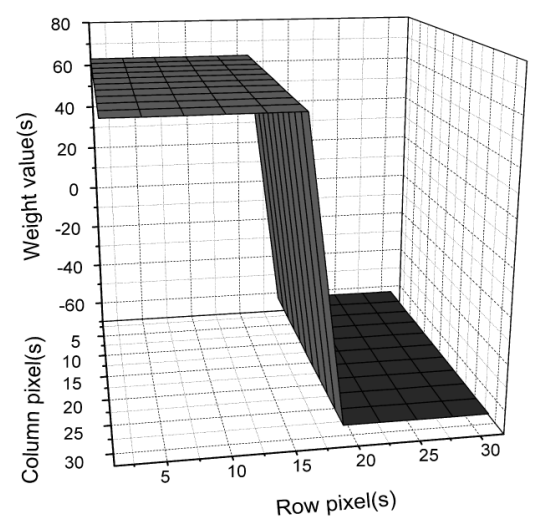

(b) 2-divided weighting function along the $\mathrm{x}$ coordinate

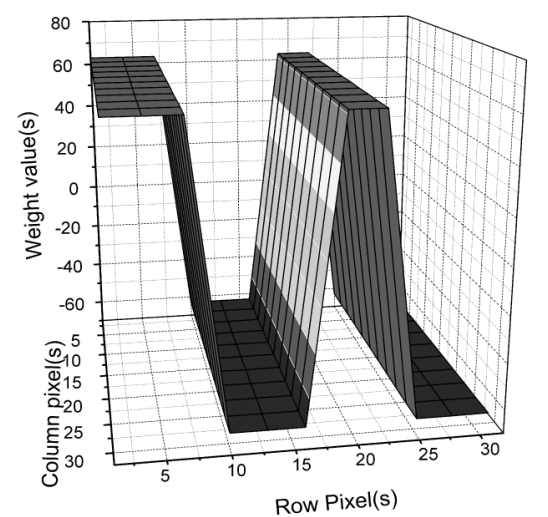

(d) 4-divided weighting function along the $\mathrm{x}$ coordinate

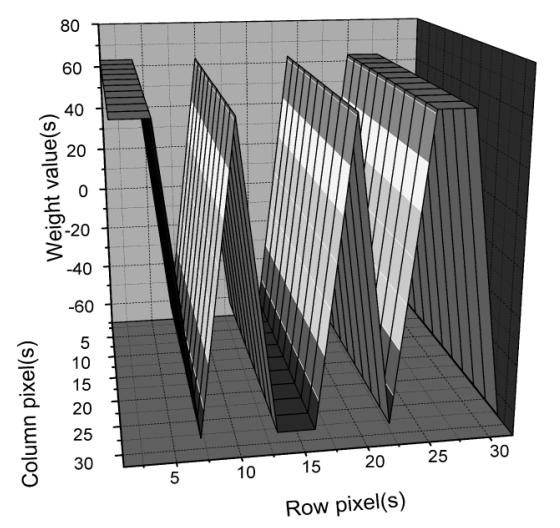

(f) 8-divided weighting function along the $\mathrm{x}$ coordinate

Fig. 13. Effects of the variable weighting function under the same moving speed

VMWF and are amplified differential output signals. Every test in the experiment to verify the characteristics of the ISFD-VMWF gives satisfactory results. From these results, the ISFD-VMWF can be adapted through changes of the weighting function to adjust spatial filter parameters for achieving adaptive measurement. Now we design the system for making small the operating system of the ISFD-VMWF using FPGA and external memories with data of the weight function. As the next subject of this study, A/D converter, address decoder, and frame memories will be integrated on the same chip.
This will enable to increase internal scanning speed and enable various applications for many kinds of physical quantity by simple setup.

(Manuscript received Dec. 2, 2004,

revised Sep. 29, 2005)

\section{References}

(1) E. Okada, H. Ehara, C. Oshio, E. Sekizuka, and H. Minamitani: "A Microscopic spatial filtering velocimeter for measurement of lymph flow in microcirculation", Proc. of 
Industrial Electronics, Contro and Intrumentation(IECON), Vol.3, pp.2375-2378 (1991)

( 2$)$ M. Nomura, M. Hori, J. Shimomura, and M. Terashima: "Velocity measurement using phase orthogonal spatial filters", IEEE Trans. Industry Appl., Vol.32, No.4, pp.796-801 (1996)

( 3 ) K.C. Michel, O.F. Fiedler, A. Richter, K. Christofori, and S. Bergeler: "A novel spatial filtering velocimeter based on a photodiode array", IEEE Trans. Instrumentation and Measurement, Vol.47, No.1, pp.299-303 (1998)

(4) M.S. Uddin, H. Inaba, Y. Itakura, Y. Yoshida, and M. Kasahara: "Adaptive computer-based spatial-filtering method for more accurate estimation of the surface velocity of debris flow", Applied Optics, Vol.38, No.32, pp.6714-6721 (1999)

(5) Y. Shinmoto, J. Takagi, K. Egawa, Y. Murata, and M. Takeuchi: "Road surface recognition sensor using an optical spatial filter", Intelligent Transportation System(ITSC) IEEE Conference on, pp.1000-1004 (1997)

(6) H.M. Go, T. Ogawa, J. Takayama, S. Ohyama, and A. Kobayashi: "Current-division-type variable spatial filtering detector with multi-valued weighting functions", Sensors and Actuators A: Physical, Vol.112, Issue 1, pp.87-93 (2004)

( 7 ) K. Kitagawa and M. Hayashi: "New Particle Counter Using Spatial Filtering Technique", 39th SICE Annual Conference, 201A-2 (2000)

(8) K. Christofori and K. Michel: "Velocimetry with spatial filters based on sensor arrays", Flow Measurement and Instrumentation, Elsevier Science Ltd, Vol.7, No.3/4, pp.265-272 (1996)

(9) M. Nakayama, T. Yamaura, and A. Kobayashi: "Optical Spatial Filter Detector for Non-Contact Measurement of Two Dimensional Velocity Vector", Trans. SICE, Vol.18, No.6, pp.70-76 (1982)

(10) A. Kobayashi, M. Nakayama, T. Yamaura, and Y. Ohkami: "Non-contact Rotational Speed Measurement and Integrated Spatial Filter Detector", Trans. SICE, Vol.15, No.1, pp.89-96 (1979)

(11) T. Ogawa: "Realization and Application of Variable Spatial Filtering Detector with Multi-Value Weighting Function", Master thesis, Tokyo Institute of Technology (1992)

(12) H.M. Go, J. Takayama, S. Ohyama, and A. Kobayashi: "Integration of Variable Spatial Filter Detector with Multi-valued Weight Values, Proceedings of International Conference on Control, Automation and Systems (ICCAS)", Vol.1, pp.921926 (2003)

(13) B. Buchanan and M. Brooke: "An experimental evaluation of error spectrum shaping applied to mixed-signal image convolutions", Circuits and Systems II: Analog and Digital Signal Processing, IEEE Transactions on ,Vol.50, Issue 12, pp.950$962(2003)$

(14) C. Mead: Analog VLSI and Neural Systems, Addison-Wesley Pub. Co. (1989)

Hyunmin Go (Non-member) received his BSEE and MSEE

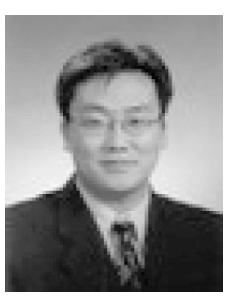
degrees in Electrical Engineering from Chonbuk National University, Korea, in 1997 and 1999, respectively. He is currently working for SAMSUNG TECHWIN CO., LTD. His basic research interests are in spatial filtering method for active measurement and its application, sensor integration (SoC) and signal processing algorithm.

Junya Takayama (Non-member) received a BE and ME de-

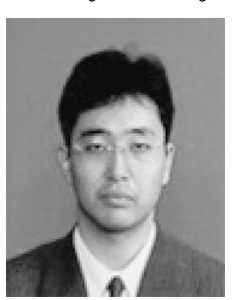
gree in Control Engineering from Tokyo Institute of Technology in 1996 and 1998 respectively. From 1998 to 2000, he was at Nissan Motor Co. Ltd., where he worked on the research and development of toroidal CVT type transmission system. Since 2000, he has been working as research associate at Tokyo Institute of Technology. His current fields of interest include the theory of signal field and its application and signal processing algorithm for fault detection.
Shinji Ohyama (Member) received a BE, ME and Doctor

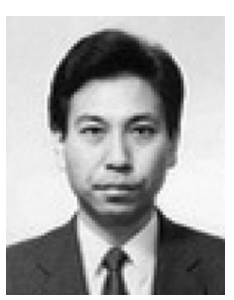
of Engineering degree in Control Engineering from Tokyo Institute of Technology in 1982, 1984 and 1998, respectively. From 1984 to 1990, he was with Hitachi Research Laboratory, Hitachi Ltd., where he worked on the research and development of micro-optic devices and an optical card system. Since 1990, he has been working at Tokyo Institute of Technology, and is presently an associate professor. His current interests include the spatial filtering method, the theory of signal field and its application and sensor network system.

Akira Kobayashi (Member) received his Doctor of Engi-

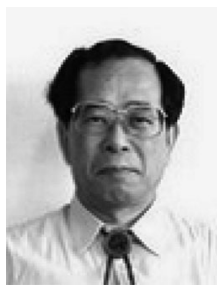
neering degree from Tokyo Institute of Technology in 1969. He is now a professor of Tokyo Institute of Technology and his current fields of interest are spatial filtering method for measurement, the sensory measurement algorithm, the theory of signal field, high SNR measurement method, and sensory evaluation using BMPC (band function model paired comparison) method. He has received SICE (The Society of Instrument and Control Engineers) Paper Awards four times. Since 1996, he has been a Fellow of SICE. 